\title{
Special Issue: Amine oxidases: structures, mechanisms and therapeutic targets
}

\author{
Andrew Holt • Darrell D. Mousseau • \\ Glen B. Baker
}

Published online: 12 June 2011

(C) Springer-Verlag 2011

On 18 July 2010, more than 60 researchers from 15 different countries gathered at the University of Alberta in Edmonton, Canada, to participate in the 14th International Amine Oxidase Workshop: AO 2010. The inaugural conference, held in 1984, was the result of the persuasive powers of Keith Tipton and Moussa Youdim, who convinced Brian Callingham to host the first official meeting of the unofficial "Amine Oxidase Club". The workshop was therefore held at Queens' College, Cambridge, and was organized by Callingham and assisted by Tipton, Youdim and Lars Oreland. Several amine oxidase meetings had been organized sporadically over a 13-year period leading up to the 1984 Cambridge workshop; the first was held 40 years ago, in Cagliari, Sardinia (1971), and four more meetings took place during the following decade, in London (1975), Midland, Michigan (1979), Hakone (1981) and Heidelberg (1982). These meetings were, for the most part, organized as unique events on behalf of institutions or companies, but the increased interest in the area following the discovery of separate MAO isoforms highlighted a need for a more regular exchange of information. Thus, the International Amine Oxidase Workshop was born, and the decision was made during the Cambridge meeting that future workshops should be held every two years.

Previous meetings have generally focused upon monoamine oxidase (MAO; EC 1.4.3.4) and semicarbazidesensitive amine oxidase/diamine oxidase (EC 1.4.3.6; recently reclassified separately as EC 1.4.3.21, primary

A. Holt $(\square) \cdot$ G. B. Baker

Edmonton, Canada

e-mail: aholt@pmcol.ualberta.ca

D. D. Mousseau

Saskatoon, Canada amine oxidase [PrAO], and EC 1.4.3.22, diamine oxidase). MAO and PrAO were covered extensively in Edmonton, with the expanding influence of MAO in various neuropathologies, as well as emerging links between PrAO and several cardiovascular and metabolic conditions, leading to much discussion. Furthermore, given the recent resurgence in interest in trace amines-neuromodulatory substrates for amine oxidases-and in their receptors, a tradition was continued by including a session covering trace amine research in the Edmonton workshop program. Discussion of the lysyl oxidase field was also introduced to the scientific program for the first time. Though lysyl oxidase (EC 1.4.3.13) is typically thought of with regard to its role in cross-linking connective tissue, significant breakthroughs in the past decade have revealed a potential involvement for this enzyme in metastatic cancers, cellular differentiation and chemotaxis. As a potential novel drug target, the development of selective therapeutics targeting lysyl oxidase would be facilitated by comparing its structure and mechanism with those of other copper-containing amine oxidases, as well as with MAOs. Similarly, the development of ideas proposing a role for PrAO in connective tissue architecture would benefit from an understanding of what has been done with lysyl oxidase in this regard, and it was with these thoughts in mind that Herb Kagan was invited to assist in introducing lysyl oxidase to the Amine Oxidase Workshop. It is hoped that these new links forged in Edmonton between related fields will be strengthened through continued participation in future workshops.

A major objective of the Edmonton meeting was to provide an opportunity for trainees and young scientists new to the field to present their work to an audience of internationally renowned experts in a relaxed and collegial setting. It is safe to say that this objective was achieved; the quality of presentations from numerous junior scientists in 
attendance was quite remarkable. Contributions from senior researchers included a Keynote Lecture on MAO structure and function by Dale Edmondson, while more than half of the invited lectures were presented by eminent scientists new to these workshops. Thus, there seems little doubt that the future of the field, and of the Amine Oxidase Workshops, looks very bright indeed.

This Special Issue of the Journal of Neural Transmission contains 16 contributions submitted by participants at $\mathrm{AO}$
2010, offering a snapshot of what was a dynamic meeting program. It is hoped that readers are encouraged by these contributions to participate in AO 2012, which will be hosted by Angelo Parini and Christian Carpéné in Toulouse in southern France.

We are grateful to Dr. Peter Riederer, Editor of the Journal of Neural Transmission, for all of his hard work that has made this Special Issue possible. 